\title{
Isoniazid and Rifampicin as Therapeutic Regimen in the Current Era: A Review
}

\author{
Sulochana Somasundaram*, Akila Ram, Laavanya Sankaranarayanan \\ Department of Biotechnology, Sri Venkateswara College of Engineering, Sriperumbudur, India \\ Email: ${ }^{*}$ sulochana@svce.ac.in
}

Received 22 December 2013; revised 25 January 2014; accepted 4 February 2014

Copyright (C) 2014 by authors and Scientific Research Publishing Inc.

This work is licensed under the Creative Commons Attribution International License (CC BY). http://creativecommons.org/licenses/by/4.0/

(c) $\underset{\mathrm{EY}}{\mathrm{B}}$ Open Access

\section{Abstract}

Tuberculosis represents one of the biggest challenges in the medical field. According to World Health Organization (WHO) Global Tuberculosis Report, 2012, there were estimated 8.7 million new TB cases worldwide while 1.4 million people died of TB. Additionally, $90 \%$ of the cases of TB are reported in developing countries, with India having the largest number of incident cases. The current treatment method includes the administration of a cocktail of drugs which includes Isoniazid (INH), Rifampicin (RIF), Ethambutol (EMB) and Pyrazinamide (PZA) which are referred to as the first line of drugs. Isoniazid and Rifampicin are currently the two most powerful anti-TB medications. The occurrences of multi-drug and extensive-drug resistant strains (MDR-TB and XDR-TB, respectively) have become a global concern and pose a serious challenge for public health management. Treatment of these resistant cases involves the usage of the second line of anti-tuberculosis drugs which are less effective than the first line and are known to cause adverse reactions or toxic side-effects. Tuberculosis research should not only focus on treatment methods but also on management of the current cases of resistance and measures to prevent an outbreak of resistant TB infection. This review outlines the mechanism of action of isoniazid and rifampicin and how resistance to these drugs emerges. We also provide a brief insight into the prevalence of $\mathrm{HIV}$ in TB patients and the challenges associated with treatment regimens in this co-infection.

\section{Keywords}

Mycobacterium tuberculosis; TB; Combination Therapy; Drug Resistance; MDR-TB; XDR-TB; Tuberculosis and HIV

\section{Introduction}

Tuberculosis, the second greatest killer worldwide after HIV/AIDS due to a single infectious organism, is a dis-

*Corresponding author. 
ease eliciting great concern in many developing countries. It is caused by Mycobacterium strains, particularly Mycobacterium tuberculosis. In 2010 alone, it is claimed that 1.4 million lives globally and 330,000 of these deaths were from India. As of 2010, India has the highest number of reported cases of TB than any other country [1]. The first line of treatment is the use of the drugs Isoniazid (INH), Rifampicin (RIF), Ethambutol (EMB) and Pyrazinamide (PZA). The Isoniazid-Rifampicin regimen, also called INHandRIF has been in use since the 1950s and is the most commonly prescribed continuation drug combination. The generally used dosage regimen is prescribed as 2HREZ/4HR3.

However, of late, cases of $M$. tuberculosis strains resistant to these drugs have been detected, complicating the treatment and prolonging the drug regimen. The occurrence of multidrug resistance has become a global concern. Studies have shown that resistance to drugs occurs due to spontaneous mutation in genes involved in the antimycobacterial actions [2]. The resistance associated mutations have been described for the first-line drugs. Researchers have shown that multi-drug resistance is developed as a consequence of accumulation of mutations, i.e., no single genetic mutation can cause the MDR phenotype. The resistance acquired over time is now called as resistance among previously treated cases; and is the more common occurrence of resistance. A combination therapy was used based on the fact that the drugs used have different modes of action and on the low statistical probabilities of spontaneous mutations that cause resistance in all drugs. INH is bactericidal against replicating bacteria while RIF is bactericidal while having a sterilizing effect. The purpose of this article is to highlight the importance of isoniazid and rifampicin in the current treatment of tuberculosis.

\section{A History of Tuberculosis}

Tuberculosis has been documented in the early days in Greece where Hippocrates recognized the disease and it was called phthisis. However, the major event in the history of tuberculosis was when Robert Koch made his presentation Die Aetiologie der Tuberculose, to the Berlin Physiological Society where he demonstrated the tubercle bacilli. He was later awarded the Noble Prize in Medicine or Physiology in 1905 for his elucidation of the etiology of tuberculosis [3]. In the early twentieth century, diagnosis of TB was done using a fluoroscopy which involved X-ray imaging of the chest. The tuberculin skin test (Mantoux test) which was designed by Mantoux in 1907 is also used to detect the presence based on a skin reaction (erythema) which is defined as positive. Bacterial diagnosis was initially done using the Ziehl-Neelsen stain with bright field microscopy. The advent of fluorescence microscopy technology gradually replaced this, as it is more efficient. Serological diagnostic techniques are used when the patients are tested smear-positive. However, this is less effective in cases where the patient has minimal disease [4].

The first milestone in the treatment of TB is generally considered to be the introduction of streptomycin antibiotic. In a study in the UK, drug resistance was seen in a five-year follow up [5]. Since single drug therapy led to the emergence of streptomycin-resistant strains, further research led to the combination treatment where streptomycin was administered along with para-aminosalicylic acid. Isoniazid, a drug which is currently in use, was first introduced in 1952 and was found to be more potent than streptomycin and para-salicylic acid. In 1967, Rifampicin was originally discovered in Italy from a new class of bacteria which produce antibiotics and was introduced for the treatment of tuberculosis along with the other anti-tuberculosis drugs. This leads to the use of combination of drugs as treatment for tuberculosis [6]. Treatment of tuberculosis can take place in hospitals (Sanatoriums) or at the home of the patient (Domiciliary Treatment). Though hospitalization is the most effective single measure in the control of tuberculosis for the individual as well as the community [7], it is difficult to accommodate large numbers of patients owing to the limited bed-space. This led to the domiciliary program where selected patients have shortened hospitalizations and the treatment was continued at the patients' residences. This became the leading practice in developing countries by the 1960s [8].

The Bacillus Calmette-Guerin (BCG) vaccine against tuberculosis was first tested in humans in 1921 and has been given to infants as a standard practice since 1974. The first randomized trial of streptomycin against pulmonary tuberculosis was carried out in 1946-1947 by the MRC Tuberculosis Research Unit, England. Currently, trials are being carried out to explore the possibility of using INH and Rifapentine (RPT) as a chemo-prophylactic in areas of high TB incidence [9]. A randomized clinical trial using these drugs was carried out in Brazil. It compared 12 weekly doses of DOT INH-RPT with 2 months of daily RIF and PZA in latent-infected subjects aged $\geq 18$ years [10]. TB was diagnosed in three INH-RPT recipients and one RIF-PZA recipient. A recent study was conducted to study the possibility of using Moxifloxacin as a primary drug along with INH, RIF and 
PZA [11] as Moxifloxacin was shown to be a potential sterilizing drug [12]. It was found that moxifloxacin significantly increased the conversion of sputum cultures to negative in patients with pulmonary tuberculosis in the Phase 2 trial and these results suggest that tuberculosis treatment can be shortened by one or several months. Advances have been made in the detection of Tuberculosis using better techniques. In a Peruvian study of 218 Peruvian children with suggestive symptoms against 238 healthy controls, Microscopic Observation Drug Susceptibility (MODS) culture increased pulmonary TB diagnostic sensitivity and speed compared with Lowenstein Jensen medium culture [13]. PCR was insufficiently sensitive or specific for routine diagnostic use, but in high risk children duplicate Gastric Aspartate PCR provided same-day identification of half of all culturepositive cases. In recent years, there has been an increase in drug-resistant strains of Mycobacteria. Many clinical trials have highlighted the importance of extensive molecular diagnostics and comprehensive phenotypic drug susceptibility testing in designing a tailored anti-tuberculosis regimen in highly resistant TB [14]-[17].

\section{Mechanism of Action of Anti-Tubercular Drugs (INH and RIF)}

\subsection{Isoniazid}

Isoniazid (also, Nicotinic Acid Hydrazide, INH or H) (Figure 1) is one of the drugs of choice for first-line treatment of tuberculosis along with Rifampicin and Pyrazinamide. It functions by blocking the production of Mycolic Acid, an essential cell wall component in the tuberculosis bacterium [18] [19]. Mycolic acid confers the bacterium with resistance to chemical damage and dehydration, and prevents the effective activity of hydrophobic antibiotics. In addition, mycolic acid allows the bacterium to grow readily inside macrophages, effectively hiding it from the host's immune system. These reasons are vital for the selection of mycolic acid as a drug target. Once circulated in the bloodstream, the isoniazid prodrug is activated via a bacterial catalase-peroxidase enzyme, encoded by KatG gene [20]. This enzyme is a hemeB containing dimer, and only one of whose domains are functional. Its role is to combat the low $\mathrm{pH}$ found during the "oxidative burst" in human phagocytes, where liberated $\mathrm{O}_{2}$ radicals are converted to $\mathrm{H}_{2} \mathrm{O}_{2}$ within the phagosome. It catalyses the formation of an activated isoniazid intermediate which binds to the InhA protein. InhA is an enoyl-acyl carrier protein reductase. This complex inhibits fatty acid synthesis and subsequently halts mycolic acid production.

The two enzymes involved in the elongation cycle of the fatty acid biosynthesis, namely enoyl-acyl carrier protein reductase (InhA) and $\beta$-ketoacyl-acyl carrier protein synthase (KasA). These are believed to be targets of the activated inhibitor [21].

Action of isoniazid is most prominent in the first week of drug administration as it acts on rapidly multiplying bacteria. On slow growing bacteria, it acts as a bacteriostatic agent.

The adverse effects of Isoniazid may result in discontinuation of treatment in some cases. The side effects can be classified astoxic, idiosyncratic and hypersensitivity reactions. Major toxic reactions are peripheral neuropathies at conventional dosage and seizures from overdosage. Idiosyncratic reactions are INH-induced lupus erythematosus, rheumatic-like syndromes and other hematologic disorders. Hypersensitivity reactions to isoniazid include hepatitis, dermatitis, fever, and haemolytic anaemia [22]. If the Mycobacterial strain is resistant to low dosage of INH (300 mg/day) in primary therapy, then a higher dosage (1 - $1.5 \mathrm{~g} /$ day) is administered to overcome the resistance. This high dosage of INH in the re-treatment of drug-resistant tuberculosis has been validated in clinical trials [23].

\subsection{Rifampicin}

Rifampicin (also 4-methyl-1-piperazinaminyl, RIF or R) (Figure 2) is a broad-spectrum lipophilic antibiotic. It inhibits bacterial RNA synthesis by binding to the $\beta$ subunit of DNA-dependent RNA polymerase, thus blocking RNA transcription [24]. The enzyme-bound drug strongly inhibits RNA chain initiation and chain elongation. The<smiles>NNC(=O)c1ccncc1</smiles> 


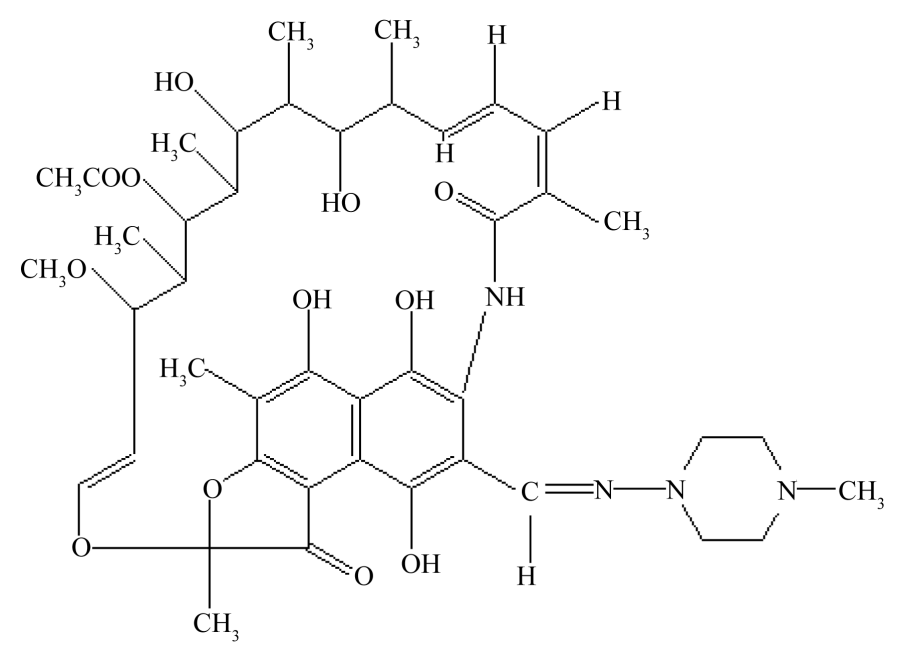

Figure 2. Structure of Rifampicin.

drug specifically inhibits the transition from synthesis of short oligoribonucleotides to full-length transcripts by a steric-occlusion mechanism. It has been documented that no treatment course shorter than 18 months has an acceptable success rate without rifampicin in the regimen [25]. In addition, rifampicin should always be used in initial anti-tuberculosis treatment, unless the patient has an isolate shown to be rifampicin resistant.

The adverse effects of Rifampicin are hepatic and immunoallergic in nature. Hepatic toxicity is dose related and has been observed mainly in patients with a history of liver disease. Patients receiving it undergo frequent liver function tests to detect liver damage as rifampicin promotes the upregulation of hepatic cytochrome P450 enzymes in the liver. Immunoallergic effects may range from minor discomforts like a cutaneous, gastrointestinal, or influenza-like syndrome to hemolyticanemia, shock, or acute renal failure [26].

\section{Emergence of Drug-Resistant Tuberculosis}

\subsection{Multi Drug-Resistant TB (MDR-TB)}

Multidrug-resistant tuberculosis (MDR-TB) occurs when the organisms show a high-level resistance to both isoniazid and rifampicin, regardless of resistance to other anti-TB drugs. The diagnosis of MDR-TB is by performing drug sensitivity tests (DST) on the isolates. DST data can also be used to identify the drug regimen. Second-line anti-tuberculosis drugs are less effective than first-line drugs and are known to cause adverse reactions more frequently. This class of anti-tuberculosis drugs includes para-aminosalicylic acid (PAS), amikacin, capreomycine, thionamide and cycloserine. New drugs such as rifapentine, levofloxacin, gatifloxacin and moxifloxacin have not yet been categorized. A retrospective study in Hong Kong compared the efficacy of two fluoroquinolones- Levofloxacin and Ofloxacin in the treatment of MDR-TB. They administered these drugs along with accompanying second-line drugs and found that Levofloxacin had a 90.0\% overall success rate as compared to $79.7 \%$ success rate of Ofloxacin [27].

\subsection{Extensively Drug-Resistant TB (XDR-TB)}

Extensively drug resistant TB (XDR-TB) is a rare type of multidrug-resistant tuberculosis (MDR-TB) in which the organisms are resistant to isoniazid and rifampicin, in addition to one fluoroquinolone and one of the secondline drugs.

Recent research has shown that Thioridazine (TDZ) may be effective in treating XDR-TB; although there needs to be more trials [28]. In another study, XDR-TB cases that were based on the DST (Drug Susceptibility Test) data and analysis (available within 31 days prior to beginning of treatment) had a better outcome when compared with XDR-TB cases in which the DST results were obtained after over 31 days of treatment [29].

\subsection{Total Drug Resistance}

Totally Drug Resistant (TDR) tuberculosis is a recent development where the Mycobacterial strains are resistant 
to both first and second line drugs. This phenomenon has been documented only in India, Iran, Italy and South Africa so far. It develops after MDR and XDR cases have been poorly handled and drug administration has been intermittent and follow-up has been irregular. The first case of TDR-TB was reported in Italy in 2006 [30] followed by Iran in 2009 [31] and India in 2012 [32]. Patients who display TDR-TB were found to be resistant to first line drugs and combinations of second line drugs when subjected to Drug Susceptibility Tests (DST). They have also been shown to display phenotypic resistance, carrying mutant versions of the KatG, rpo $\beta$, gyrA, rrsand InhA genes.

As there are no drugs currently prescribed for cases with this degree of severity, it has been stressed by the Centres of Disease Control (CDC), USA that patients who have been tested positive for TDR-TB should be allowed access to promising drugs that have demonstrated in-vitro activity against $M$. tuberculosis.

\section{Mode of Resistance}

While the modes of resistance for of the anti-tuberculosis drugs haven't fully been understood and characterized, some of the reasons which have been documented are described here.

\subsection{Isoniazid}

Monoresistance to isoniazid is the most common mutation in $M$. tuberculosis strains, leading to drug resistance. In addition, the development of INH resistance is more complex than Rifampicin [33]. In vivo, INH is activated by the enzyme catalase peroxidase encoded by the $k a t G$ gene. Studies show that mutations in this gene are seen in approximately 50\% of INH-resistant strains [34]. Of these, the most common mutation is the Ser315Thr mutation which causes an enzyme with reduced activity but with high-level resistance to isoniazid. In a study of 85 INHresistant strains from global sources, amino acid 315 (Ser) was found to be mutated in 49 (58\%) isolates [35]. The other less-frequent mutations yield resistance of varying levels. The abundance of KatG315 amino acid changes can be attributed at a study in which the activities of wild-type KatG were compared with a mutant. The catalase activity of the Ser315Thr mutant was reduced 6- fold, whereas the peroxidase activity was only decreased $<2$-fold. The Ser315Thr mutant is a competent catalase-peroxidase that has reduced ability to metabolize INH. This is important as it allows the bacterium to detoxify anti-bacterial radicals while simultaneously reducing the conversion of INH to its prodrug [36].

In case of low-level isoniazid resistance, mutations were found in promoter regions of inhA, acpM and kasA. The mutations of these are thought to cause over-expression of these proteins; thus rendering isoniazid to be ineffective [37].

\subsection{Rifampicin}

Rifampicin resistance occurs only after an initial resistance to another drug such as isoniazid. Thus, it could be used as a diagnosis for MDR TB.

Isolates which are resistant to rifampicin have a mutation that alters the sequence of a 27-amino-acid region of the $\beta$ subunit of the RNA polymerase enzyme. Studies have shown that over $95 \%$ of the rifampicin resistant strains have a particular mutation in the $r p o \beta$ gene in the $81 \mathrm{bp}$ region [34].

High level resistance most commonly occurs (65\% - 86\%) due to alterations in codon526 or codon531. The most common amino acid substitutions were Ser531Leu (42\%) and His526Tyr (23\%) [38] (Figure 3). Mutations in codons 511, 516, 518 and 522 have shown to cause low-level resistance to rifampicin and rifapentinbut are still susceptible to rifalazyn and rifabutin. Susceptibility tests were conducted globally in locations such as Korea, Japan, Germany and Mozambique. The frequency of altered codons were analysed and compared with the global results [39]-[41]. It was shown that geographic differences do not account for Rifampicin resistance, i.e., the frequency of the altered codons 526 or 531 cannot be said to be concentrated in any geographical location.

Various molecular diagnostic techniques exist for determining RIF resistance. They include genotypic tests like DNA sequencing, polymerase chain reaction single strand conformation polymorphism (PCR-SSCP), real-time PCR and Line probe Assays and phenotypic tests such as Luciferase reporter phage assay,BACTEC MGIT 960 system and phage amplified biological assay (PhaB) [42]. DNA sequencing is considered to be the gold standard for molecular diagnostics and has been shown to be more effective in determining Rifampicin resistance as compared to other tests [43]. 


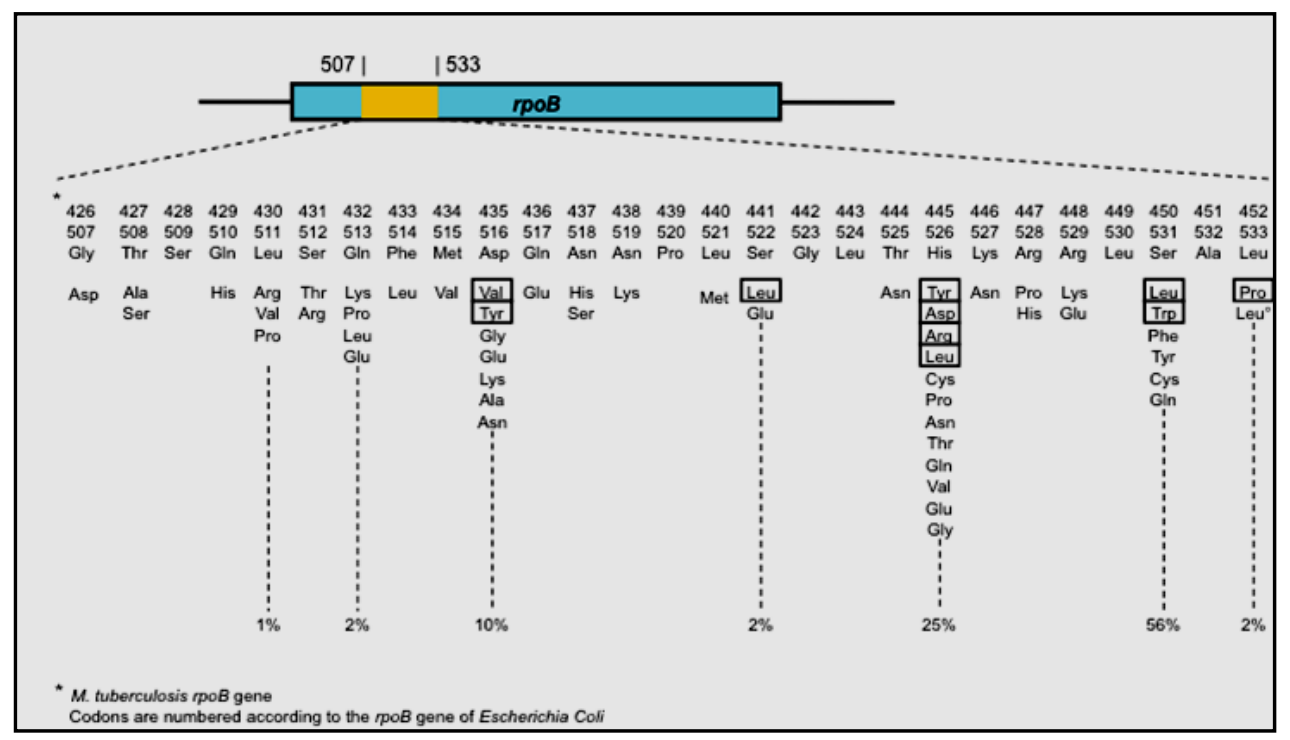

Figure 3. Missense mutations of the $r p o B$ gene associated with resistance to Rifampicin in $M$. tuberculosis. Ref: [44].

As RIF binds to the $\beta$-subunit of the RNA polymerase, the region surrounding it (codon 507-533) is considered a resistance hotspot, called Rifampicin Resistance Determining Region (RRDR). Mutations within this hotspot are most likely to cause RIF resistance [44].

Table 1 summarises the commonly used anti-tuberculosis drugs, their site of action and the gene responsible for resistance development.

\section{Emergence of Drug Resistance to INH and RIF Regimens}

\subsection{Non-Compliance Leads to Drug Resistance}

One of the mechanisms by which MDR strains develop is due to non-compliance of the patients, i.e., they do not complete the entire course of treatment.

At the beginning of INH and RIF regimen, Isoniazid has a high bactericidal activity. This condition will favour the mutant's resistance to INH alone. If the patient stops taking the regimen in $2-3$ days, it is likely that the bacterial population will grow again with an increase in number of INH-resistant mutants. Rifampicin is known to selectively kill some of the dormant population of the bacteria; thus effectively creating a monotherapy-like condition. Sub-inhibitory concentrations of RIF will cause re-growth leading to selection towards those cells resistant to the drug [45].

When the treatment regimen is specified for a certain number of months, it is done on the basis of the fact that there are cycles of bactericidal activity and re-growth of the dormant bacilli. When patients stop taking the drugs before the completion of the drug regimen, it gives a chance for the dormant bacilli to regrow. But this population will be highly favoured toward resistant strains. This can occur in widely-spaced combination therapy as well [46]. This defines the rationale behind the importance of supervision to ensure completion of the treatment.

The factors that could contribute to non-compliance include: complexity and duration of regimen, difficult access to health care centre particularly in rural areas, adverse side effects, lack of attaching importance to regimen, the feeling of having no more symptoms to treat, treatment for other conditions like AIDS.

\subsection{Monotherapy Can Lead to Resistance}

When a single drug is given to a TB patient, it often happens that the bacterial population which was initially sensitive to the drug becomes resistant to it. This is due to the fact that once a single drug is administered; it favours the selection of mutants resistant to it. Thus, the growth of previously dormant bacilli will begin to exhibitresistance. Isoniazid resistance is frequently encountered among the M. tuberculosis population. Monotherapylike conditions in a combination therapy can also induce resistance. Isoniazid is generally the primary resistance as 
Table 1. Common anti-tubercular drugs and their associated resistances.

\begin{tabular}{ccc}
\hline Drug & Site of Action & Mutation in gene \\
\hline Isoniazid & Mycolic acid synthesis & katG, inhA, acpM, kasA \\
Rifampicin & RNA synthesis & Rpo $\beta$ \\
Streptomycin & Ribosomal S2 protein & $r p s L$ \\
Pyrazinamide & Pyrazinamidase enzyme & pncA \\
Ethambutol & Arabinosyltransferase enzyme & embB \\
Kanamycin & $16 S$ rRNA & Rrs \\
Ethionamide & Mycolic acid synthesis & etaA \\
\hline
\end{tabular}

INH acts first on the multiplying bacteria, in the initial few days of combination therapy. Rifampicin resistance can later occur when the bactericidal cycle shifts to the dormant bacilli.

The main reason for the combination therapy is that giving four drugs at once will reduce the possibility of resistance development. Thus, even though there are new drugs being researched, they are always given along H\&R to ensure that monoresistant bacilli do not emerge as a result of the treatment [47] [48].

\section{Tuberculosis and HIV}

Immunocompromised HIV patients have between 20 - 37 times greater risk of developing tuberculosis as compared to individuals without HIV infection. The co-infection with tuberculosis complicates the treatment regimen, resulting in non-responsiveness of the individual to Anti-retroviral therapy (ART). Moreover, in the presence of HIV, diagnosis of TB cannot be made based only on the conventional diagnostic methods, vis. Sputum smear and Tuberculin test, as they do not provide adequate diagnostic help. Comprehensive clinical and radiological investigations have to be conducted in order to diagnose TB at immunosuppressed states [49].

HIV infection is associated with both an increased incidence of TB and altered clinical symptoms. HIV progression is characterized by the inability to react to a skin test (cutaneous anergy) and impaired tissue containment of mycobacteria, leading to widespread dissemination of the bacterium [50]. Observational studies on patients with advanced HIV have reported a higher risk of acquired rifampicin resistance when highly intermittent regimens are used in these patients [51]. Factors that have been associated with higher failure and relapse rates along with increased mortality during treatment and follow-up are: severe immunosuppression, highly intermittent rifampicin administration, lack of rifampicin in the regimen used and duration of treatment of $<6$ months.

\section{Antagonistic Activity of INH and RIF towards Anti-Retroviral Drugs}

Drugs used in the treatment of tuberculosis interfere with the anti-retroviral drugs, lowering their efficacy. Rifampicin induces the CYP-3A enzyme system in the liver and intestinal wall, thereby increasing the metabolism of the antiretroviral drugs- Protease Inhibitors (PI) and non-nucleoside reverse transcriptase inhibitors (NNRTIs). When rifampicin and antiretroviral drugs are administered together, decreased trough levels (i.e. the lowest level that a medicine is present in the body) may result, leading to therapeutic failure. Peripheral neuropathy which can be due to HIV can be further aggravated by Isoniazid [52]. Clinical trials in HIV-infected persons have shown that the incidence of TB can be reduced by $40 \%-60 \%$ by preventive therapy (chemoprophylaxis) [53]. The standard chemoprophylactic regimen is Isoniazid for 12 months.

As there is presently no national policy regarding TB preventive therapy for HIV positive persons in India, Highly Active Anti Retroviral Therapy (HAART) can be used for MDR-HIV patients. A Brazilian population study reported an $80 \%$ reduction in TB incidence in HAART-treated HIV-infected patients compared to ARTnaïve patients [54] [55]. Through further clinical studies and a rigorous monitoring of HIV status, it is possible to develop an HAART drug regimen suitable for the Indian population.

\section{Tuberculosis Treatment in the Current Era}

Taking into consideration the rate at which drug resistances in tuberculosis are steadily increasing, it is the need of the hour that novel drugs are introduced and the treatment duration is shortened. In this regard, new classes of drugs have been developed such as fluoroquinolones, nitroimidazoles, oxazolindiones, etc. A combination therapy 
containing the right cocktail of these drugs can be administered to the patient depending on his level of resistance to other drugs. It has been shown that Moxifloxacin and Gatifloxacin were proposed as a combination therapy in the condition of low-level quinolone resistance owing to their low mean MIC [56]. Another novel compound that is gaining widespread attention is PA-824. It is a niroimidazone that has been shown to be active against both replicating and non-replicating bacteria [57]. Usage of this new compound resulted in a much higher bactericidal activity $(0 \mathrm{CFU} / \mathrm{ml})$ as compared to treatment with rifampicin (5 CFU/ml) and pyrazinamide (7 CFU/ml) [58]. In a study of phase II clinical trials in South Africa, the combination therapy PaMZ, which consists of PA-824, moxifloxacin and pyrazinamide killed more than $99 \%$ of TB bacteria within 14 days, thus making it potentially suitable for treating both drug sensitive and multidrug resistant tuberculosis [59].

In a recent press release, TB Alliance has licensed CSIR India to begin Phase $2 \mathrm{~b}$ trials for a treatment regimen containing PA-824, Moxifloxacin and Pyrazinamide (PaMZ) at low cost. This regimen has been shown to be effective in shortening treatment duration, including some drug-resistant forms of TB [60] (Figure 4).

Other promising drugs that are in various stages of clinical trials are: Bedaquiline, SQ109, Sutezolid, Linezolid, Metronidazole and AZD5847 among many others. SQ109 is anethylenediamine that could be used in cases of phenotypic resistance to rifampicin and isoniazid in a combination therapy [61]. Bedaquiline is a diarylquinalone whose addition to the standard regimen for multi-drug resistant tuberculosis resulted in quicker conversion to negative sputum culture [62]. An important aspect in clinical trials involving any new compound is that it is always introduced in combination with the established anti-mycobaterial drugs: Isoniazid, Rifampicin and Pyrazinamide. Hence, notwithstanding the development of new anti-tubercular drugs and rising multi-drug resistances, Isoniazid and Rifampicin remain a major component of modern tuberculosis treatment regimens.

\section{Summary}

Drug-resistant TB poses a significant threat to humans and is a global cause for concern. It is said that one in three persons have dormant tuberculosis bacteria and the person develops TB when the bacteria are no longer dormant. According to Espinal et al., 2001, MDR-TB poses a serious problem in Eastern Europe (Estonia, Latvia and Russia), highly populated countries like Indian and China (which accounts for $39 \%$ of the TB cases) and countries in Africa. MDR-TB is associated with higher rates of failure and death, so countries should adopt programs that would be effective such as the Directly Observed Therapy (DOTS) and DOTS plus+ of the World Health Organization. While usage of second line drugs is considered, it should be done in accordance with established tuberculosis control programs. Although Rifampicin is the primary drug responsible for killing the bacilli, studies and research in the Americas where Rifampicin is eliminated in the continuation phase of the therapy has shown markedly low number of resistant cases [63]. With such statistics, is essential to not only treat TB, MDR-TB and XDR-TB, but also work on preventing the further emergence of drug resistant bacteria. The combination therapy has been internationally approved, and this along with strict adherence to the drug regimen will help the Directly Observed Therapy (DOTS) and DOTS plus+ programs in treating MDR-TB and also the non-compliant patients. Further studies in assessing the mutation involved in resistance (particularly KatG) using new technology will help in understanding the different dimensions of the pathogenesis which will ultimately be useful for new drug

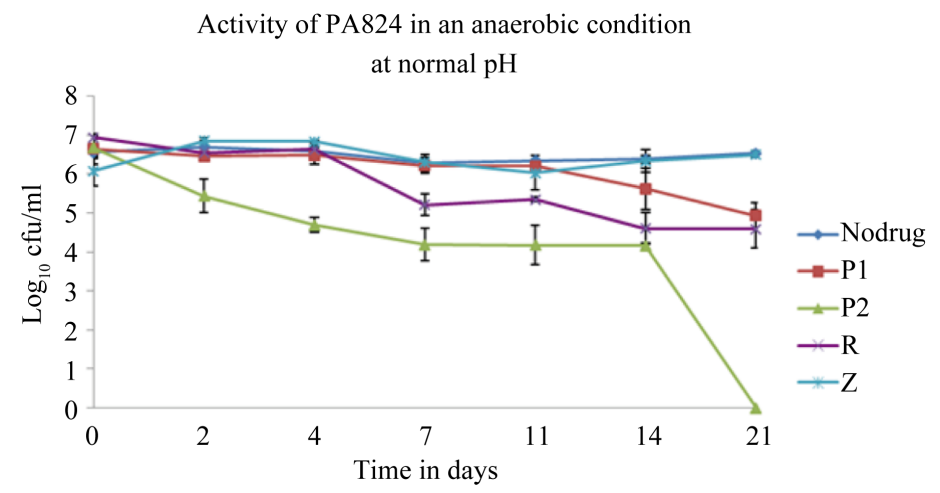

Figure 4. Bactericidal activity of PA-824 on Mycobacterium tuberculosis H37 RV under anaerobic condition. 
targets and preventive intervention. Studies on the hypermutable state which is induced by certain physiological conditions would provide more insights in preventing resistance in the strains.

However, it is of prime importance to prevent an epidemic of multi drug resistance strains to ensure that it is not transmitted to the community. The measures to manage multi drug resistance will be successful only when there is judicial usage of the first line drugs while discouraging use of second-line drugs. There should also be measures taken to prevent the transmission of the mutant strains in the community.

Thus, it should be noted that the two most potent drugs are isoniazid and rifampicin and there are no substitutes for this currently. The combination therapy is important because it can help to avoid emergence of drug resistance as in the case of monotherapy. The focus should be on wise usage of these first line drugs along with adhering to tuberculosis treatment protocols so that the probabilities for mutation and drug resistance are reduced.

In the current era of tuberculosis management, novel therapeutic compounds have been introduced along with Isoniazid and Rifampicin. Thus, although there are increasing cases of worldwide resistance to Isoniazid and Rifampicin, they are an indispensable asset in the treatment and management of tuberculosis.

\section{Acknowledgements}

The authors thank Sri Venkateswara College of Engineering, Sriperumbudur, for their support in preparing the manuscript.

\section{References}

[1] WHO (1997) Anti-Tuberculosis Drug Resistance in the World: The WHO/IUATLD Global Project on Anti-Tuberculosis Drug Resistance Surveillance 1994-1997. World Health Organization, Geneva, WHO/TB/97.229.

[2] Somoskovi, A., Parsons, L.M. and Salfinger, M. (2001) The Molecular Basis of Resistance to Isoniazid, Rifampin, and Pyrazinamide in Mycobacterium tuberculosis. Respiratory Research, 2, 164-168. http://dx.doi.org/10.1186/rr54

[3] Daniel, T.M. (2006) The History of Tuberculosis. Respiratory Medicine, 100, 1862-1870. http://dx.doi.org/10.1016/j.rmed.2006.08.006

[4] Mitchison, D.A. (2005) The Diagnosis and Therapy of Tuberculosis during the Past 100 Years. American Journal of Respiratory and Critical Care Medicine, 171, 699-706. http://dx.doi.org/10.1164/rccm.200411-1603OE

[5] Dawson, J.J.Y., et al. (1966) A 5-Year Study of Patients with Pulmonary Tuberculosis in a Concurrent Comparison of Home and Sanatorium Treatment for One Year with Isoniazid plus PAS. Bulletin of the World Health Organization, 34, 533.

[6] Mitchison, D.A. (2005) Drug Resistance in Tuberculosis. The European Respiratory Journal: Official Journal of the European Society for Clinical Respiratory Physiology, 25, 376-379. http://dx.doi.org/10.1183/09031936.05.00075704

[7] Kane, A., Brigham, H. and Pollak, S. (1953) Domiciliary Treatment of Pulmonary Tuberculosis. Chest, 23, 51-531. http://dx.doi.org/10.1378/chest.23.5.518

[8] Tuberculosis Chemotherapy Centre (1973) A Controlled Comparison of Two Fully Supervised Once-Weekly Regimens in the Treatment of Newly Diagnosed Pulmonary Tuberculosis. Tubercle, 54, 23-45. http://dx.doi.org/10.1016/0041-3879(73)90013-5

[9] Sterling, T.R., Villarino, M.E., Borisov, A.S., Shang, N., Gordin, F., Bliven-Sizemore, E. and Chaisson, R.E. (2011) Three Months of Rifapentine and Isoniazid for Latent Tuberculosis Infection. New England Journal of Medicine, 365, 2155-2166. http://dx.doi.org/10.1056/NEJMoa1104875

[10] Schechter, M., Zajdenverg, R. and Falco, G. (2006) Weekly Rifapentine/Isoniazid or Daily Rifampin/Pyrazinamide for Latent Tuberculosis in Household Contacts. American Journal of Respiratory and Critical Care Medicine, 173, 922926. http://dx.doi.org/10.1164/rccm.200512-19530C

[11] Conde, M.B., Efron, A., Loredo, C., De, G.R.M., Graça, N.P., Cezar, M.C. and Ram, M. (2009) Moxifloxacin in the Initial Therapy of Tuberculosis: A Randomized, Phase 2 Trial. Lancet, 373, 1183-1189. http://dx.doi.org/10.1016/S0140-6736(09)60333-0

[12] Sulochana, S., Mitchison, D.A., Kubendiren, G., Venkatesan, P. and Paramasivan, C.N. (2009) Bactericidal Activity of Moxifloxacin on Exponential and Stationary Phase Cultures of Mycobacterium tuberculosis. Journal of Chemotherapy, 21, 127-134. http://dx.doi.org/10.1179/joc.2009.21.2.127

[13] Oberhelman, R.A., Soto-Castellares, G., Gilman, R.H., Caviedes, L., Castillo, M.E., Kolevic, L. and Evans, C.A. (2010) Diagnostic Approaches for Paediatric Tuberculosis by Use of Different Specimen Types, Culture Methods, and PCR: A Prospective Case-Control Study. The Lancet Infectious Diseases, 10, 612-620. http://dx.doi.org/10.1016/S1473-3099(10)70141-9 
[14] Feasey, N.A., Pond, M., Coleman, D., Solomon, A.W., Cosgrove, C.A., Delgado, R. and Harrison, T. (2011) Moxifloxacin and Pyrazinamide Susceptibility Testing in a Complex Case of Multidrug-Resistant Tuberculosis (Case Study). The International Journal of Tuberculosis and Lung Disease, 15, 417-420.

[15] Adetifa, I.M., Ota, M.O., Jeffries, D.J., Lugos, M.D., Hammond, A.S., Battersby, N.J. and Hill, P.C. (2013) Interferon- $\gamma$ ELISPOT as a Biomarker of Treatment Efficacy in Latent Tuberculosis Infection: A Clinical Trial. American Journal of Respiratory and Critical Care Medicine, 187, 439-445. http://dx.doi.org/10.1164/rccm.201208-1352OC

[16] Boehme, C.C., Nabeta, P., Hillemann, D., Nicol, M.P., Shenai, S., Krapp, F. and Perkins, M.D. (2010) Rapid Molecular Detection of Tuberculosis and Rifampin Resistance. New England Journal of Medicine, 363, 1005-1015. http://dx.doi.org/10.1056/NEJMoa0907847

[17] Narayanan, S., Swaminathan, S., Supply, P., Shanmugam, S., Narendran, G., Hari, L. and Narayanan, P.R. (2010) Impact of HIV Infection on the Recurrence of Tuberculosis in South India. Journal of Infectious Diseases, 201, 691-703. http://dx.doi.org/10.1086/650528

[18] Lei, B.F. and Wei, C.-J. (2000) Action Mechanism of Antitubercular Isoniazid. The Journal of Biological Chemistry, 275, 2520-2526. http://dx.doi.org/10.1074/jbc.275.4.2520

[19] Vilchèze, C., Morbidoni, H.R., Weisbrod, T.R., Iwamoto, H., Kuo, M., Sacchettini, J.C. and Jacobs, W.R. (2000) Inactivation of the inhA-Encoded Fatty Acid Synthase II (FASII) Enoyl-Acyl Carrier Protein Reductase Induces Accumulation of the FASI End Products and Cell Lysis of Mycobacterium smegmatis. Journal of Bacteriology, 182, 4059-4067. http://dx.doi.org/10.1128/JB.182.14.4059-4067.2000

[20] Whitney, J.B. and Wainberg, M.A. (2002) Isoniazid, The Frontline of Resistance in Mycobacterium tuberculosis. McGill Journal of Medicine, 6, 114-123.

[21] Timmins, G.S. And Deretic, V. (2006) Mechanisms of Action of Isoniazid. Molecular Microbiology, 62, $1220-1227$. http://dx.doi.org/10.1111/j.1365-2958.2006.05467.x

[22] Goldman, A.L. and Braman, S.S. (1972) Isoniazid: A Review with Emphasis on Adverse Effects. Chest, 62, 71-77. http://dx.doi.org/10.1378/chest.62.1.71

[23] Moulding, T.S. (1981) Should Isoniazid Be Used in Retreatment of Tuberculosis Despite Acquired Isoniazid Resistance? The American Review of Respiratory Disease, 123, 262.

[24] Calvori, C., Frontali, L., Leoni, L. and Tecce, G. (1965) Effect Ofrifamycin on Protein Synthesis. Nature, 207, 417-418. http://dx.doi.org/10.1038/207417a0

[25] Mitchison, D.A. (2000) Role of Individual Drugs in the Chemotherapy of Tuberculosis. The International Journal of Tuberculosis and Lung Disease, 4, 796-806.

[26] Grosset, J. and Leventis, S. (1983) Adverse Effects of Rifampin. Review of Infectious Diseases, 5, S440-S446. http://dx.doi.org/10.1093/clinids/5.Supplement_3.S440

[27] Yew, W.W., Chan, C.K., Leung, C.C., Chau, C.H., Tam, C.M., Wong, P.C. and Lee, J. (2003) Comparative Roles of Levofloxacin and Ofloxacin in the Treatment of Multidrug-Resistant Tuberculosis: Preliminary Results of a Retrospective Study From Hong Kong. Chest, 124, 1476-1481. http://dx.doi.org/10.1378/chest.124.4.1476

[28] van Ingen, J., van der Laan, T., Amaral, L., Dekhuijzen, R., Boeree, M.J. and van Soolingen, D. (2009) In Vitro Activity of Thioridazine against Mycobacteria. International Journal of Antimicrobial Agents, 34, 190-191. http://dx.doi.org/10.1016/j.ijantimicag.2009.02.015

[29] Sotgiu, G., Ferrara, G., Matteelli, A., Richardson, M.D., Centis, R., et al. (2009) Epidemiology and Clinical Management of XDR-TB: A Systematic Review by TBNET. European Respiratory Journal, 33, 871-881. http://dx.doi.org/10.1183/09031936.00168008

[30] Migliori, G.B., De Iaco, G., Besozzi, G., Centis, R. and Cirillo, D.M. (2007) First Tuberculosis Cases in Italy Resistant to All Tested Drugs. Eurosurveillance, 12, 3194.

[31] Velayati, A.A., Masjedi, M.R., Farnia, P., Tabarsi, P., Ghanavi, J., Ziazarifi, A.H. and Hoffner, S.E. (2009) Emergence of New Forms of Totally Drug-Resistant Tuberculosis bacilli: Super Extensively Drug-Resistant Tuberculosis or Totally Drug-Resistant Strains in Iran. Chest, 136, 420-425. http://dx.doi.org/10.1378/chest.08-2427

[32] Udwadia, Z.F., Amale, RA., Ajbani, K.K. and Rodriguez, C. (2012) Totally Drug-Resistant Tuberculosis in India. Clinical Infectious Diseases, 54, 579-581. http://dx.doi.org/10.1093/cid/cir889

[33] Rouse, D.A. and Morris, S.L. (1995) Molecular Mechanisms of Isoniazid Resistance in Mycobacterium tuberculosis and M. bovis. Infection and Immunity, 63, 1427-1433.

[34] Ramaswamy, S. and Musser, J.M. (1998) Molecular Genetic Basis of Antimicrobial Agent Resistance in Mycobacterium tuberculosis. Tubercle and Lung Disease: The Official Journal of the International Union against Tuberculosis and Lung Disease, 79, 3-29. http://dx.doi.org/10.1054/tuld.1998.0002

[35] Musser, J.M. (1995) Antimicrobial Agent Resistance in Mycobacteria: Molecular Genetic Insights. Clinical Microbi- 
ology Reviews, 8, 496-514.

[36] Wengenack, N.L., Uhl, J.R., Amand, A.L.S., Tomlinson, A.J., Benson, L.M., Naylor, S. and Rusnak, F. (1997) Recombinant Mycobacterium tuberculosis KatG (S315T) Is a Competent Catalase-Peroxidase with Reduced Activity toward Isoniazid. Journal of Infectious Diseases, 176, 722-727. http://dx.doi.org/10.1086/514096

[37] Zhang, Y., Heym, B., Allen, B., Young, D. and Cole, S. (1992) The Catalase-Peroxidase Gene and Isoniazid Resistance of Mycobacterium tuberculosis. Nature, 358, 591-593. http://dx.doi.org/10.1038/358591a0

[38] Musser, J.M. (1995) Antimicrobial Agent Resistance in Mycobacteria: Molecular Genetic Insights. Clinical Microbiology Reviews, 8, 496-514.

[39] Caugant, D.A., Sandven, P., Eng, J., Jeque, J.T. and Tønjum, T. (1995) Detection of Rifampin Resistance among Isolates of Mycobacterium tuberculosis from Mozambique. Microbial Drug Resistance, 1, 321-326. http://dx.doi.org/10.1089/mdr.1995.1.321

[40] Rinder, H., Dobner, P., Feldmann, K., Rifai, M., Bretzel, G., Rüsch-Gerdes, S. and Löscher, T. (1997) Disequilibria in the Distribution of $r p o B$ Alleles in Rifampicin-Resistant M. tuberculosis Isolates from Germany and Sierra Leone. Microbial Drug Resistance, 3, 195-197. http://dx.doi.org/10.1089/mdr.1997.3.195

[41] Kim, S.J., Bai, G.H. and Hong, Y.P. (1997) Drug-Resistant Tuberculosis in Korea, 1994. The International Journal of Tuberculosis and Lung Disease, 1, 302-308.

[42] Mani, C., Selvakumar, N., Kumar, V., Narayanan, S. and Narayanan, P.R. (2003) Comparison of DNA Sequencing, PCR-SSCP and PhaB Assays with Indirect Sensitivity Testing for Detection of Rifampicin Resistance in Mycobacterium Tuberculosis. The International Journal of Tuberculosis and Lung Disease: The Official Journal of the International Union against Tuberculosis and Lung Disease, 7, 652-659.

[43] Telenti, A., Imboden, P. and Marchesi, F. (1993) Detection of Rifampicin-Resistance Mutations in Mycobacterium Tuberculosis. Lancet, 341, 647-651. http://dx.doi.org/10.1016/0140-6736(93)90417-F

[44] Pierre-Audigier, C. and Gicquel, B. (2011) The Contribution of Molecular Biology in Diagnosing Tuberculosis and Detecting Antibiotic Resistance, 1-47. http://www.moleculartb.org/gb/pdf/doc/Revue.pdf

[45] Mitchison, D.A. (1998) How Drug Resistance Emerges as a Result of Poor Compliance during Short Course Chemotherapy for Tuberculosis. The International Journal of Tuberculosis and Lung Disease, 2, 10-15.

[46] Burman, W.J., Cohn, D.L., Rietmeijer, C.A., Judson, F.N., Sbarbaro, J.A. and Reves, R.R. (1997) Noncompliance with Directly Observed Therapy for Tuberculosis: Epidemiology and Effect on the Outcome of Treatment. Chest, 111, 1168-1173. http://dx.doi.org/10.1378/chest.111.5.1168

[47] Blomberg, B., Spinaci, S., Fourie, B. and Laing, R. (2001) The Rationale for Recommending Fixed-Dose Combination Tablets for Treatment of Tuberculosis. Bulletin of the World Health Organization, 79, 61-68.

[48] Paramasivan, C.N. (1998) Overview on Drug Resistant Tuberculosis in India. Lung India, 16, 21-28.

[49] Deivanayagam, C.N., Rajasekaran, S., Raja, K., Chandrasekar, C., Palanisamy, S., Dinesh, A.S., Jothivel, G., et al. (2001) Clinico-Radiological Spectrum of Tuberculosis among HIV-Seropositives: A Tambaram Study. Indian Journal of Tuberculosis, 48, 7-10.

[50] Swaminathan, S. (2008) HIV and Tuberculosis in India. Journal of Biosciences, 33, 527-537.

[51] Chaisson, R.E., Schecter, G.F., Theuer, C.P., Rutherford, G.W., Echenberg, D.F. and Hopewell, P.C. (1987) Tuberculosis in Patients with the Acquired Immunodeficiency Syndrome: Clinical Features, Response to Therapy, and Survival. American Review of Respiratory Disease, 136, 570-574. http://dx.doi.org/10.1164/ajrccm/136.3.570

[52] Shafer, R.W. and Edlin, B.R. (1996) Tuberculosis in Patients Infected with Human Immunodeficiency Virus: Perspective on the Past Decade. Clinical Infectious Diseases, 22, 683-704. http://dx.doi.org/10.1093/clinids/22.4.683

[53] Chakraborty, A.K. (2004) Epidemiology of Tuberculosis: Current Status in India. The Indian Journal of Medical Research, 120, 248-276.

[54] Miranda, A., Morgan, M., Jamal, L., Laserson, K., Barreira, D., Silva, G. and Santos, J. (2007) Impact of Antiretroviral Therapy on the Incidence of Tuberculosis: The Brazilian Experience, 1995-2001. PloS ONE, 2, e826. http://dx.doi.org/10.1371/journal.pone.0000826

[55] Rabahi, M.F., Rodrigues, A.B., Queiroz de Mello, F., de Almeida Netto, J.C. and Kritski, A.L. (2002) Noncompliance with Tuberculosis Treatment by patients at a Tuberculosis and AIDS Reference Hospital in Midwestern Brazil. The Brazilian Journal of Infectious Diseases: An Official Publication of the Brazilian Society of Infectious Diseases, 6, 6373. http://dx.doi.org/10.1590/S1413-86702002000200002

[56] Somasundaram, S. and Paramasivan, N.C. (2006) Susceptibility of Mycobacterium tuberculosis Strains to Gatifloxacin and Moxifloxacin by Different Methods. Chemotherapy, 52, 190-195. http://dx.doi.org/10.1159/000093486

[57] Tyagi, S., Nuermberger, E., Yoshimatsu, T., Williams, K., Rosenthal, I., Lounis, N. and Grosset, J. (2005) Bactericidal 
Activity of the Nitroimidazopyran PA-824 in a Murine Model of Tuberculosis. Antimicrobial Agents and Chemotherapy, 49, 2289-2293. http://dx.doi.org/10.1128/AAC.49.6.2289-2293.2005

[58] Somasundaram, S., Anand, R.S., Venkatesan, P. and Paramasivan, C.N. (2013) Bactericidal Activity of PA-824 against Mycobacterium tuberculosis under Anaerobic Conditions and Computational Analysis of Its Novel Analogues against Mutant Ddn Receptor. BMC Microbiology, 13, 218. http://dx.doi.org/10.1186/1471-2180-13-218

[59] Diacon, A.H., Dawson, R., Von Groote-Bidlingmaier, F., Symons, G., Venter, A., Donald, P.R., van Niekerk, C., Everitt, D., Winter, H., Becker, P., Mendel, C.M. and Spigelman, M.K. (2012) 14-Day Bactericidal Activity of PA-824, Bedaquiline, Pyrazinamide, and Moxifloxacin Combinations: A Randomised Trial. Lancet, 380, 986-993. http://dx.doi.org/10.1016/S0140-6736(12)61080-0

[60] TB Alliance (2013) TB Alliance Licenses Late-Stage TB Program to CSIR-OSDD. http://www.tballiance.org/newscenter/view-brief.php?id=1076

[61] Sacksteder, K.A., Protopopova, M., Barry, C.E., Andries, K. and Nacy, C.A. (2012) Discovery and Development of SQ109: A New Antitubercular Drug with a Novel Mechanism of Action. Future Microbiology, 7, 823-837. http://dx.doi.org/10.2217/fmb.12.56

[62] Diacon, A.H., Pym, A., Grobusch, M., Patientia, R., Rustomjee, R., Page-Shipp, L., Neeley, D.F.M., et al. (2009) The Diarylquinoline TMC207 for Multidrug-Resistant Tuberculosis. The New England Journal of Medicine, 360, 23972405. http://dx.doi.org/10.1056/NEJMoa0808427

[63] Espinal, M., Laszlso, A., Simonsen, L., Boulahbal, F., Jae Kim, S., Reniero, A., Hoffner, S., Rieder, H.L., Binkin, N., Dye, C., Williams, R. and Raviglione, M.C. (2001) Global Trends in Resistance to Anti-Tuberculosis Drugs. The New England Journal of Medicine, 344, 1294-1303. http://dx.doi.org/10.1056/NEJM200104263441706 